Published in final edited form as:

Cardiol Clin. 2017 May ; 35(2): 223-230. doi:10.1016/j.ccl.2016.12.004.

\title{
Psychosocial factors and hypertension: A review of the literature
}

\author{
Adolfo G. Cuevas, PhD, \\ Cancer Prevention Postdoctoral Fellow, Department of Social and Behavioral Sciences, Harvard \\ T.H. Chan School of Public Health, Boston, MA
}

David R. Williams, PhD, MPH, and

Florence Sprague Norman \& Laura Smart Norman Professor of Public Health, Department of Social and Behavioral Sciences, Harvard T.H. Chan School of Public Health, Boston, MA

Michelle A. Albert, MD, MPH

CeNter for the StUdy of AdveRsiTy and CardiovascUlaR DiseasE [NURTURE Center], University of California, San Francisco, Division of Cardiology, Department of Medicine, San Francisco, CA

\section{Keywords}

Hypertension; Psychosocial factors; Health disparities; Race/ethnicity; Review

\section{Introduction}

Hypertension is a pervasive problem in the United States, with approximately a third of Americans reporting being diagnosed with hypertension by their physicians or taking antihypertensive medicine. ${ }^{1}$ Hypertension is an important risk factor for a variety of health conditions, such as cardiovascular disease, stroke, and kidney failure. ${ }^{1}$ Nevertheless, this burden is unevenly distributed in society, with Blacks having the highest prevalence of hypertension compared to their White counterparts. ${ }^{1}$ Despite improvements in increasing the awareness and treatment of hypertension, racial/ethnic differences in hypertension persist.

Growing evidence points to multiple psychological and social factors as contributors to the onset of and trajectory of hypertension. Psychosocial factors that induce emotional stress can evoke a physiological response meditated in part by activation of the sympathetic nervous system, inflammation, and the hypothalamic-pituitary-adrenal axis. ${ }^{2,3}$ Repeated activation of this system can result in failing to return to resting blood pressure (BP) levels.

CORRESPONDING AUTHOR Correspondence concerning this article should be addressed to David R. Williams at Harvard T. H. Chan School of Public Health, Department of Social and Behavioral Sciences. 677 Huntington Ave, 6th Floor, Boston, MA 02115. dwilliam@hsph.harvard.edu. Adolfo G. Cuevas, Harvard T.H. Chan School of Public Health, Department of Social and Behavioral Sciences, Landmark Center, Rm 428a, 401 Park Drive, Boston, MA 02215. acuevas @ hsph.harvard.edu;. Michelle A. Albert, CeNter for the StUdy of AdveRsiTy and CardiovascUlaR DiseasE [NURTURE Center], University of California, San Francisco, Division of Cardiology, Department of Medicine, 505 Parnassus Avenue, M1177, San Francisco, CA 94143. michelle.albert@ucsf.edu.

Publisher's Disclaimer: This is a PDF file of an unedited manuscript that has been accepted for publication. As a service to our customers we are providing this early version of the manuscript. The manuscript will undergo copyediting, typesetting, and review of the resulting proof before it is published in its final citable form. Please note that during the production process errors may be discovered which could affect the content, and all legal disclaimers that apply to the journal pertain.

DISCLOSURE STATEMENT

The Authors have nothing to disclose. 
Psychosocial factors, such as hostility and job strain, have been found to be associated with higher circulating levels of catecholamines, higher cortisol levels, and increased BP over time. ${ }^{4}$

Prior reviews have identified a number psychosocial indicators as potential risk factors for the onset and progression of hypertension. ${ }^{2,5}$ The purpose of this review is to provide an overview of recent findings related to major psychosocial factors and hypertension. Due to space constraints, emerging work about psychosocial factors (e.g., personality, sleep quality ${ }^{5}$ ) cannot be fully discussed. Nevertheless, in presenting the major factors, we highlight gaps in the extant literature that contribute to our limited understanding of the social determinants of the persistent racial/ethnic hypertension disparities and discuss directions for future research.

\section{Socioeconomic status}

Socioeconomic status (SES) has long been identified as a risk factor for hypertension. A review by Spruill suggests a complex interaction of social, psychological, and behavioral factors contributing to unequal distribution of diseases. ${ }^{2}$ Compared to their high SES peers, individuals of low SES are more likely to lack socio-political power and economic resources thereby resulting occupancy of less health enhancing educational, occupational, residential and recreational environments. ${ }^{6}$ These factors lead to differential exposures to stressors (e.g., unemployment, crime and violence) and fewer resources (e.g., recreation and physical activity) to cope with accumulation of stressors that combine to contribute to greater risk of hypertension. ${ }^{2}$ In a recent meta-analysis, multiple indicators of SES (i.e., income, occupation, and education) were associated with an increased risk of hypertension. ${ }^{7}$

SES and race/ethnicity are closely intertwined. ${ }^{2}$ Contemporary racial/ethnic categories simultaneously capture unmeasured confounding for biological factors associated with ancestral history and geographic origins, factors linked to current and earlier psychological, social, physical, chemical environmental exposures, as well as biological adaptation to these exposures. ${ }^{8}$ Racial/ethnic differences in SES are large and persistent, and likely contribute to racial/ethnic differences in hypertension. Recent national data reveal Black households earn 59 cents and Hispanics ones 70 cents for every dollar of income that Whites receive; moreover, Blacks have only 6 cents and Hispanics 7 cents for every dollar of wealth that Whites have. ${ }^{6}$

Additionally, because of the persistence of a residual association between race and hypertension after controlling for modifiable risk factors and SES, other unmeasured explanatory factors likely contribute to hypertension disparities. ${ }^{9}$ Research suggests that a lifecourse perspective provides insight into the prolonged impact that early SES can have on blood pressure. While most studies taking a lifecourse perspective are cross-sectional, they suggest that the accumulation of stress due to SES positioning likely promotes healthdamaging effects later in life. For instance, low childhood SES and childhood adversity are associated with higher risk of hypertension. ${ }^{10,11}$ Slopen et al. find that a positive neighborhood context may modify the relationship between childhood adversity and cumulative biological risk in adulthood. ${ }^{12}$ Importantly, as evidence suggests that racial/ ethnic minorities have higher cumulative stress than Whites, ${ }^{13}$ further research is needed to 
examine the extent to which race/ethnicity may moderate the association between the accumulation of stressors over the lifecourse and hypertension.

\section{Race/ethnicity-related Stress}

Discrimination-Growing attention is paid to the ways in which race/ethnicity-related aspects of social experience may adversely affect health, such as discrimination. ${ }^{9}$ Discrimination can erode an individual's health through negative psychological and physiological responses and untoward health maintenance and behaviors. ${ }^{14}$ Nevertheless, the literature on discrimination and hypertension is riddled with inconsistent findings, partly due to measurement issues and the relative lack of longitudinal studies. ${ }^{9,15}$ For example, research from the Jackson Heart Study found that lifetime discrimination and the burden of discrimination were each modestly associated with increased hypertension prevalence. ${ }^{16}$ However, no association was observed between hypertension and a measure of current everyday discrimination. It is plausible that reports of current exposure to minor instances of discrimination might be related to short-term measured BP change, whereas lifetime measures might more aptly capture the cumulative effect of discriminatory exposure on blood pressure risk over time. ${ }^{16}$ Not surprisingly, chronic discrimination is more consistently associated with ambulatory blood pressure than with resting clinic BP. ${ }^{17}$

Pathways through which discrimination might affect hypertension risk are multiple.

Whereas, prior research indicates that Blacks were more likely than Whites to have a blunted blood pressure decline during sleep, emerging studies reveal that exposure to discrimination contributes to the elevated levels of blood pressure and lack of BP dip among Blacks at night. ${ }^{14}$ Sleep disturbances are also related to hypertension risk. Moreover, a recent review indicates that discrimination is positively associated with reported sleep difficulties, insomnia, and fatigue, thereby influencing hypertension risk. ${ }^{18}$ In other research, threat of exposure to discrimination vs. actual exposure also affects hypertension risk, possibly via heightened vigilance. ${ }^{19}$ Experiences of discrimination have also been associated with lower levels of adherence and follow-up for multiple health conditions, and if this pattern is also true of hypertension, it could contribute to greater severity and poorer course of hypertension. ${ }^{14}$ In light of the above, future studies should consider the multiple dimensions of discrimination and conditions that adversely affect blood pressure. ${ }^{17}$

Goal-striving stress-There are other more novel race-related risk factors that may also play a role. Goal-striving stress is a measure of thwarted aspirations. It is typically operationalized as the discrepancy between aspirations and achievement, weighted by the subjective probability of success and the level of disappointment experienced if goals are not reached. ${ }^{20}$ Analyses of a large national sample revealed that higher levels of goal-striving stress was associated with the prevalence of self-reported hypertension among White Americans, African Americans, and Caribbean Blacks and race/ethnicity did not moderate the relationship between goal-striving stress and hypertension. ${ }^{20}$ Longitudinal research is needed to enhance our understanding of how the timing of the development of goal-striving stress and how exposure and response to it may influence the onset and course of hypertension disparities. Relatedly, John Henryism, an active predisposition to succeed against all odds, is associated with high blood pressure among persons who lack the 
resources to facilitate success. ${ }^{21}$ Research is needed to better understand the conditions under which psychological risk factors and coping mechanisms such as goal-striving stress and John Henryism can affect the development and course of hypertension and the behavioral and biological mechanisms through which these processes occur. ${ }^{21}$

Internalized racism-Self-stereotyping (or internalized racism) has also been identified as another mechanism by which racism might adversely affect health. Limited research indicates positive association between internalized racism and multiple health outcomes including overweight/obesity and blood pressure. ${ }^{14}$ Other data show that internalized racism interacts with discrimination to predict elevated risk of hypertension, ${ }^{22}$ such that, in a sample of 91 African American men, elevated hypertension risk was only evident for African American men who scored high both on discrimination and anti-Black bias.

Stereotype threat is a term describing the activation of negative stereotypes in members of a stigmatized group that creates reactions, anxieties and expectations which adversely affect psychological functioning and health. ${ }^{23}$ For example, in the work by Blascovich et al., physiological arousal triggered by stereotype threat is associated with greater increases in blood pressure among African Americans than in European American students. ${ }^{24}$ Stereotype threat is also purported to trigger increases in anxiety and impairment of decision-making and self-regulation processes in a manner that increases aggressive behavior and overeating. ${ }^{25}$ Stereotype threat may be indirectly related to hypertension through weight changes and modulation of neural hormonal system. ${ }^{26,27}$ Finally, stereotype threat can lead affected persons to delay seeking needed medical care, and have poor adherence to recommended therapies and generally poor patient-provider relationships. ${ }^{14}$

\section{Other Sources of Stress}

Occupational Stress-Previous research suggests that working conditions that induce stress are associated with increased risk of hypertension. ${ }^{2,28}$ Occupational stressors can include hostile work environments (e.g., threatened, bullied, or harassed by anyone while you were on the job), work insecurity (e.g., worried about becoming unemployed), time pressures, work hazards, and other work conditions (e.g., sedentary tasks, uncontrollable tasks). ${ }^{28}$ High job strain/stress (high job demand and low job control) is independently associated with hypertension, ambulatory blood pressure elevation, and cardiovascular disease risk. ${ }^{29-31}$

Indeed, occupations with high job demand and low job control (e.g., servers and clerks) are commonly overrepresented among ethnic minorities. ${ }^{32}$ However, the relationship between job strain and hypertension have been mixed, particularly for Blacks. For instance, low decisional control has been found to be related to increased BP reactivity among Blacks. ${ }^{33,34}$ However, a recent study using Health and Retirement Study data, found no association between high job strain (as well as workplace discrimination) and hypertension among African Americans. ${ }^{35}$ Considering the paucity of epidemiological studies examining the relationship between work-related stressors and hypertension among racial/ethnic minority workers, these results require replication using national datasets. Furthermore, other job strain domains, especially those that may be more salient for ethnic minorities (such as 
stressors linked to unemployment and underemployment, job conflict, or financial strain due to low wages) should be explored in this area of research. ${ }^{35}$

An important direction for future research is to more comprehensively assess the broad range of stressors that might affect hypertension risk. For example, with regards to work stress, studies need to go beyond assessing high demand and low control and assess conditions in work environments that are likely to vary by race such as physical and chemical environmental hazards and occupational stressors linked to injury risk. ${ }^{8}$ As research studies have assessed only a few domains of stressors, systematic attention needs to be given to understanding the contribution of a broader range of living conditions that could increase hypertension risk, including incarceration that disproportionately affects Blacks.

For example, the CARDIA study found that incarceration was associated with increased risk of incident hypertension three years later and greater end organ damage related to hypertension. ${ }^{36}$ Incarceration is a type of stressful experience that exacerbates social disadvantage by placing many on a pathway of low education, poor job prospects, and low income. These examples highlight the need to fully characterize all aspects of the social context that can negatively affect hypertension risk.

\section{Emotional States}

High levels of anxiety and depression symptoms are common in adults, often co-morbid with chronic illnesses such as hypertension and can have deleterious effects on individual health and quality of life. A meta-analysis of prospective studies found that depressive symptoms predict a $42 \%$ increased risk of hypertension. ${ }^{37}$ Similarly to the findings for depressive symptoms, a meta-analysis of prospective studies found that anxiety symptoms were an independent risk factor for incident hypertension. ${ }^{38}$ However, many studies are limited by the lack of control for confounding factors, such as risk behaviors and related psychological factors (e.g., anger), which may attenuate observed relationships between these emotional states and hypertension. Future research should adjust for all related factors to accurately assess the independent contribution of depression and anxiety to hypertension.

Consistent with prior research, recent evidence continues to document that anger and hostility are associated with increased hypertension risk. ${ }^{39}$ For example, using data from the Heart Strategies Concentrating On Risk Evaluation, researchers noted that high levels of hostility are associated with an attenuated nocturnal decline in BP among Blacks and Whites. ${ }^{40} \mathrm{~A}$ majority of research in this area has been limited to White men, ${ }^{39}$ which also limits our understanding of racial and gender differences in levels of exposures to these risk factors and the extent to which the effects of such exposures vary by social group. In addition, as noted by Trudel-Fitzgerald and colleagues, few prospective studies with up-todate methodology have been conducted within the last decade. ${ }^{39}$ However, longitudinal analyses from the Jackson Heart Study shows that African Americans with high anger-out (expressed rather than repressed anger) scores have a 20\% increased risk of BP progression compared to African Americans with low anger-out scores. ${ }^{41}$ Ideally, prospective studies are needed to identify the independent effect of anger (both experience and expression) on blood pressure in diverse population samples. 
Trudel-Fitzgerald and colleagues introduced two emerging psychosocial factors that should be considered within the larger literature of emotional states and hypertension, positive psychological well-being and emotion regulation. ${ }^{39}$ Growing evidence suggests that positive psychological well-being (PPWB), such as optimism, life satisfaction, and emotional vitality, is positively associated with hypertension and cardiovascular health risk factors (e.g., physical activity). ${ }^{39,42}$ However, the link between PPWB and hypertension has not been well established due to methodological and measurement issues (e.g., the heterogeneity of hypertension measurements). ${ }^{39}$ Similarly, while vitality predicts hypertension onset, ${ }^{43}$ further research using a broader range of PPWB domains are needed to fully understand the influence of PPWB on hypertension. Finally, emotion regulation, which refers to the monitoring of one's emotional experiences and responses, may also influence physical health, including hypertension risk, but research in this area is limited by lack of diversity and longitudinal studies. ${ }^{39}$

\section{Social Relationships}

Social relationships serve as sources of emotional support (e.g., empathy), informational support and instrumental support. These positive aspects of social ties have been shown to directly enhance health and to reduce the negative effects of stressful experiences on health by enhancing individuals' capacity to cope with stress. These processes are also likely to affect hypertension risk. ${ }^{2,5}$ For example, a study using National Health Interview Survey data found that both emotional support and social integration were independently associated with decreased odds of hypertension. ${ }^{44}$ The study also suggested that emotional support and social integration appeared to buffer the adverse effects of low SES on hypertension. ${ }^{44}$ Yet, the interrelationships between race/ethnicity, hypertension, and social relationships have not been clearly elucidated. The limited available research in this area indicates that race/ethnic differences in hypertension are reduced among those who receive social support, compared to those who do not receive any form of support. ${ }^{45}$ Although this study did not find an interaction effect between marital status and ethnicity in predicting hypertension, the potential contribution of marital status, a key component of social relationships, deserves more concerted attention in research on hypertension. Marital status, similar to other measures of social ties, can have both positive and negative effects on health, particularly hypertension. ${ }^{2}$ However, inadequate attention has been given to examining the association between marital relationships, and other types of social ties and blood pressure among ethnic minorities and their potential contribution to hypertension disparities. This is especially important given that blacks are less likely to be married than whites and it is currently unclear if other social relationships compensate for the lower levels of marital ties among Blacks.

\section{Other Research Priorities}

The role of genetics in racial/ethnic differences in hypertension remains as an ongoing debate. While evidence suggests that polymorphisms in the APOL 1 gene may contribute to hypertension propensity in Blacks, ${ }^{46}$ socio-environmental influences are critical. For example, research demonstrates that while Blacks in the U.S. have higher rates of hypertension than Whites in European countries such as Sweden and Italy, they have lower prevalence levels than Whites in other countries such as Germany and Finland.$^{47}$ Increasing 
evidence also suggests that epigenetic modifications, due to, for instance, childhood development or chemical exposure, are important contributors to the development of hypertension. ${ }^{48}$ However, there are only a limited number of studies of epigenetic changes linked to hypertension risk factors, and only blood rather than the effector tissues have been examined, limiting our understanding of the contribution of DNA methylation to high blood pressure. ${ }^{48}$ Future studies should consider examining both blood methylation and effector tissues to more fully understand the contribution of epigenetics to hypertension disparities.

Another issue of concern relates to the lack of attention to or the assumption of nonheterogeneity within ethnic groups. Studies of Blacks tend to cluster immigrant African and Caribbean Blacks with US-born Blacks, regardless of acculturation status and country-oforigin. Whites are also viewed as monolithic with little attention given to recent Eastern European immigrants or groups from North Africa and the Middle East that are grouped into the official 'White' category in the U.S. Similarly, studies cluster Hispanics, regardless of acculturation status, race, and country-of-origin, potentially diluting the measurement of the differential impact of environment on hypertension risk. Greater acculturation is associated with increased hypertension risk, independent of age, gender, race/ethnicity, education, smoking, alcohol, physical activity, body mass index, and diabetes. ${ }^{49}$ Additionally, there is disconcertingly little research about the determinants of hypertension risk in America's indigenous populations (Native Hawaiians and other Pacific Islanders and American Indians and Alaskan Natives), despite a higher prevalence of hypertension in these groups compared to other ethnic groups. ${ }^{50}$ An enhanced understanding of psychosocial risks and resources associated with the historical and contemporary conditions in which these groups live, learn, work, play and worship can facilitate the identification of culturally sensitive intervention strategies for hypertension. ${ }^{51}$

Finally, while other psychosocial factors, such as sleep disturbances, were not covered in depth in this article, their exclusion does not diminish the value of their inclusion in future research. For example, sleep quality and certain personality factors (e.g., neuroticism) may contribute to hypertension risk. ${ }^{5}$ However, similar to several other factors discussed, much of the evidence comes from studies of Whites, which restricts our understanding as to how they may contribute to existing hypertension disparities.

\section{Psychosocial Interventions}

Currently, lifestyle modification remains the most effective interventions to reduce hypertension risk. Lifestyle modification, including increasing physical activity, reducing alcohol intake, and reducing sodium intake, through programs such as Dietary Approaches to Stop Hypertension (DASH), reduces hypertension risk. ${ }^{52}$ While lifestyle modification is important, especially for Blacks and Hispanics who have high rates of overweight/obesity and physical inactivity, making lifestyle changes is very challenging for disadvantaged populations in the absence of comprehensive efforts to address the underlying social conditions that give rise to the risk behaviors in the first place. ${ }^{53}$ Socially disadvantaged groups face a multitude of stressors, coupled with limited resources, which can limit the effectiveness of interventions that are narrowly focused on the behavior without attention to the social conditions that initiate and sustain them. ${ }^{54}$ 
Stress and depression management/interventions should be complementary additions to lifestyle modification. Evidence suggests that stress-reducing interventions, such as Transcendental Meditation, decreases diastolic and systolic blood pressures. ${ }^{55,56}$ To address race-related stressors, such as racial discrimination, Lewis and colleagues highlight promising interventions that can reduce the effects of discrimination on health, such as religious involvement, values affirmation, forgiveness, and racism countermarketing. ${ }^{57}$

Interventions addressing SES disparities at the neighborhood level are also important. ${ }^{8}$ Racial/ethnic and SES segregation in particular can increase the risk of morbidity and mortality through limited socioeconomic mobility, restrained access to health resources, exposure to toxins and environmental stressors (e.g., violence), and weakened neighborhood social capital. ${ }^{8}$ Further research priority needs to be given to understanding the complex influence of poverty and racial segregation on health. Policies and interventions that improve neighborhood and housing quality, increase household income and improve education can help to improve the health of socially-disadvantaged populations. ${ }^{54}$ For example, randomized housing interventions (e.g., Moving to Opportunity) showed that moving poor residents from high-poverty public housing apartments to lower-poverty neighborhood environments, with no health interventions, reduced obesity and diabetes risk 10 to 15 years later, ${ }^{56}$ an action that likely also influences blood pressure outcomes.

\section{Conclusion}

Understanding hypertension disparities remains an important and complex issue in human health. There is a great need for further research to better understand and effectively address the role of psychosocial factors in the disproportionate burden of hypertension on racial/ ethnic minorities. Taking a lifecourse perspective that captures the accumulation of risks over time may help address some of the questions about the effect of psychosocial factors on hypertension risk that still puzzle researchers. In addition, future research should address methodological limitations of the existing literature including identifying and controlling for a broad set of core confounding factors, using appropriately accurate measures of BP (e.g., self-reported hypertension vs. ambulatory blood pressure), and doing more longitudinal research. Addressing such issues could help disentangle existing complexities and generate new insights that can potentially greatly improve the effectiveness of interventions that seek to reduce hypertension disparities.

\section{Bibliography}

1. Mozaffarian D, Benjamin EJ, Go AS, et al. Executive Summary: Heart Disease and Stroke Statistics — 2016 Update. Circulation. 2016; 133(4):447-454. doi:10.1161/CIR.0000000000000366. [PubMed: 26811276]

2. Spruill TM. Chronic Psychosocial Stress and Hypertension. Curr Hypertens Rep. 2010; 12(1):10 16. doi:10.1007/s11906-009-0084-8. [PubMed: 20425153]

3. Black PH, Garbutt LD. Stress, inflammation and cardiovascular disease. J Psychosom Res. 2002; 52(1):1-23. [PubMed: 11801260]

4. Everson-Rose SA, Lewis TT. Psychosocial Factors and Cardiovascular Diseases. Annu Rev Public Health. 2005; 26(1):469-500. doi:10.1146/annurev.publhealth.26.021304.144542. [PubMed: 15760298] 
5. Cuffee Y, Ogedegbe C, Williams NJ, Ogedegbe G, Schoenthaler A. Psychosocial Risk Factors for Hypertension: An Update of the Literature. Curr Hypertens Rep. 2014; 16(10):483. doi:10.1007/ s11906-014-0483-3. [PubMed: 25139781]

6. Williams DR, Priest N, Anderson N. Understanding Associations between Race, Socioeconomic Status and Health: Patterns and Prospects. Health Psychol Off J Div Health Psychol Am Psychol Assoc. 2016; 35(4):407-411. doi:10.1037/hea0000242.

7. Leng B, Jin Y, Li G, Chen L, Jin N. Socioeconomic status and hypertension: a meta-analysis. J Hypertens. 2015; 33(2):221-229. doi:10.1097/HJH.0000000000000428. [PubMed: 25479029]

8. Williams DR, Mohammed SA, Leavell J, Collins C. Race, socioeconomic status, and health: complexities, ongoing challenges, and research opportunities. Ann N Y Acad Sci. 2010; 1186:69101. doi:10.1111/j.1749-6632.2009.05339.x. [PubMed: 20201869]

9. Dolezsar CM, McGrath JJ, Herzig AJM, Miller SB. Perceived racial discrimination and hypertension: a comprehensive systematic review. Health Psychol. 2014; 33(1):20-34. doi:10.1037/ a0033718. [PubMed: 24417692]

10. James SA, Van Hoewyk J, Belli RF, Strogatz DS, Williams DR, Raghunathan TE. Life-Course Socioeconomic Position and Hypertension in African American Men: The Pitt County Study. Am J Public Health. 2006; 96(5):812-817. doi:10.2105/AJPH.2005.076158. [PubMed: 16571689]

11. Crowell JA, Davis CR, Joung KE, et al. Metabolic pathways link childhood adversity to elevated blood pressure in midlife adults. Obes Res Clin Pract. Nov.2015 doi:10.1016/j.orcp.2015.10.009.

12. Slopen N, Non A, Williams DR, Roberts AL, Albert MA. Childhood Adversity, Adult Neighborhood Context, and Cumulative Biological Risk for Chronic Diseases in Adulthood. Psychosom Med. 2014; 76(7):481-489. doi:10.1097/PSY.0000000000000081. [PubMed: 25077427]

13. Sternthal MJ, Slopen N, Williams DR. Racial Disparities in Health: The Impact of MultiDimensional Measures of Race/Ethnicity on the Self-Reported Health Status of Latinos. Bois Rev Soc Sci Res Race. 2011; 8(1):95-113. doi:10.1017/S1742058X11000087.

14. Williams DR, Mohammed SA. Racism and Health I: Pathways and Scientific Evidence. Am Behav Sci. 2013; 57(8) doi:10.1177/0002764213487340.

15. Cuffee YL, Hargraves JL, Allison J. Exploring the association between reported discrimination and hypertension among African Americans: a systematic review. Ethn Dis. 2012; 22(4):422-431. [PubMed: 23140072]

16. Sims M, Diez-Roux AV, Dudley A, et al. Perceived discrimination and hypertension among African Americans in the Jackson Heart Study. Am J Public Health. 2012; 102(Suppl 2):S258 265. doi:10.2105/AJPH.2011.300523. [PubMed: 22401510]

17. Brondolo E, Love EE, Pencille M, Schoenthaler A, Ogedegbe G. Racism and hypertension: a review of the empirical evidence and implications for clinical practice. Am J Hypertens. 2011; 24(5):518-529. [PubMed: 21331054]

18. Slopen N, Lewis TT, Williams DR. Discrimination and sleep: a systematic review. Sleep Med. 2016; 18:88-95. doi:10.1016/j.sleep.2015.01.012. [PubMed: 25770043]

19. Hicken MT, Lee H, Morenoff J, House JS, Williams DR. Racial/Ethnic Disparities in Hypertension Prevalence: Reconsidering the Role of Chronic Stress. Am J Public Health. 2014; 104(1):117-123. doi:10.2105/AJPH.2013.301395. [PubMed: 24228644]

20. Sellers SL, Neighbors HW, Zhang R, Jackson JS. The impact of goal-striving stress on physical health of white Americans, African Americans, and Caribbean blacks. Ethn Dis. 2012; 22(1):2128. [PubMed: 22774305]

21. Bennett GG, Merritt MM, III JJS, et al. Stress, coping, and health outcomes among AfricanAmericans: a review of the John Henryism hypothesis. Psychol Health. 2004; 19(3):369-383. doi: $10.1080 / 0887044042000193505$.

22. Chae DH, Nuru-Jeter AM, Adler NE. Implicit racial bias as a moderator of the association between racial discrimination and hypertension: a study of Midlife African American men. Psychosom Med. 2012; 74(9):961-964. doi:10.1097/PSY.0b013e3182733665. [PubMed: 23107842]

23. Steele CM. A threat in the air. How stereotypes shape intellectual identity and performance. Am Psychol. 1997; 52(6):613-629. [PubMed: 9174398] 
24. Blascovich J, Spencer SJ, Quinn D, Steele C. African Americans and high blood pressure: the role of stereotype threat. Psychol Sci. 2001; 12(3):225-229. [PubMed: 11437305]

25. Inzlicht M, Kang SK. Stereotype threat spillover: how coping with threats to social identity affects aggression, eating, decision making, and attention. J Pers Soc Psychol. 2010; 99(3):467-481. doi: 10.1037/a0018951. [PubMed: 20649368]

26. Meyer N, Richter SH, Schreiber RS, et al. The unexpected effects of beneficial and adverse social experiences during adolescence on anxiety and aggression and their modulation by genotype. Front Behav Neurosci. 2016:97. doi:10.3389/fnbeh.2016.00097. [PubMed: 27303275]

27. Rana S, Pugh PC, Katz E, et al. Independent effects of early-life experience and trait aggression on cardiovascular function. Am J Physiol - Regul Integr Comp Physiol. Jun.2016 ajpregu.00505.2015. doi:10.1152/ajpregu.00505.2015.

28. Rosenthal T, Alter A. Occupational stress and hypertension. J Am Soc Hypertens JASH. 2012; 6(1):2-22. doi:10.1016/j.jash.2011.09.002. [PubMed: 22024667]

29. Babu GR, Jotheeswaran AT, Mahapatra T, et al. Is hypertension associated with job strain? A metaanalysis of observational studies. Occup Environ Med. 2014; 71(3):220-227. doi:10.1136/ oemed-2013-101396. [PubMed: 24142979]

30. Landsbergis PA, Dobson M, Koutsouras G, Schnall P. Job strain and ambulatory blood pressure: a meta-analysis and systematic review. Am J Public Health. 2013; 103(3):e61-71. doi:10.2105/ AJPH.2012.301153.

31. Slopen N, Glynn RJ, Buring JE, Lewis TT, Williams DR, Albert MA. Job strain, job insecurity, and incident cardiovascular disease in the Women's Health Study: results from a 10-year prospective study. PloS One. 2012; 7(7):e40512. doi:10.1371/journal.pone.0040512. [PubMed: 22815754]

32. Buchanan S, Vossenas P, Krause N, et al. Occupational injury disparities in the US hotel industry. Am J Ind Med. 2010; 53(2):116-125. doi:10.1002/ajim.20724. [PubMed: 19593788]

33. Thomas KS, Nelesen RA, Ziegler MG, Bardwell WA, Dimsdale JE. Job Strain, Ethnicity, and Sympathetic Nervous System Activity. Hypertension. 2004; 44(6):891-896. doi:10.1161/01.HYP. 0000148499.54730.0d. [PubMed: 15534076]

34. Curtis AB, James SA, Raghunathan TE, Alcser KH. Job strain and blood pressure in African Americans: the Pitt County Study. Am J Public Health. 1997; 87(8):1297-1302. [PubMed: 9279264]

35. Mezuk B, Kershaw KN, Hudson D, Lim KA, Ratliff S. Job Strain, Workplace Discrimination, and Hypertension among Older Workers: The Health and Retirement Study. Race Soc Probl. 2011; 3(1):38-50. doi:10.1007/s12552-011-9041-7. [PubMed: 22096475]

36. Wang E, Pletcher M, Lin F, et al. Incarceration, incident hypertension, and access to health care: Findings from the coronary artery risk development in young adults (cardia) study. Arch Intern Med. 2009; 169(7):687-693. doi:10.1001/archinternmed.2009.26. [PubMed: 19364998]

37. Meng L, Chen D, Yang Y, Zheng Y, Hui R. Depression increases the risk of hypertension incidence: a meta-analysis of prospective cohort studies. J Hypertens. 2012; 30(5):842-851. doi: 10.1097/HJH.0b013e32835080b7. [PubMed: 22343537]

38. Pan Y, Cai W, Cheng Q, Dong W, An T, Yan J. Association between anxiety and hypertension: a systematic review and meta-analysis of epidemiological studies. Neuropsychiatr Dis Treat. 2015; 11:1121-1130. doi:10.2147/NDT.S77710. [PubMed: 25960656]

39. Trudel-Fitzgerald C, Gilsanz P, Mittleman MA, Kubzansky LD. Dysregulated Blood Pressure: Can Regulating Emotions Help? Curr Hypertens Rep. 2015; 17(12):1-9. doi:10.1007/ s11906-015-0605-6.

40. Mezick EJ, Matthews KA, Hall M, et al. Low Life Purpose and High Hostility are Related to an Attenuated Decline in Nocturnal Blood Pressure. Health Psychol Off J Div Health Psychol Am Psychol Assoc. 2010; 29(2):196-204. doi:10.1037/a0017790.

41. Ford CD, Sims M, Higginbotham JC, et al. Psychosocial Factors Are Associated With Blood Pressure Progression Among African Americans in the Jackson Heart Study. Am J Hypertens. 2016; 29(8):913-924. doi:10.1093/ajh/hpw013. [PubMed: 26964661]

42. Boehm JK, Kubzansky LD. The heart's content: the association between positive psychological well-being and cardiovascular health. Psychol Bull. 2012; 138(4):655-691. doi:10.1037/a0027448. [PubMed: 22506752] 
43. Trudel-Fitzgerald C, Boehm JK, Kivimaki M, Kubzansky LD. Taking the tension out of hypertension: a prospective study of psychological well being and hypertension. J Hypertens. 2014; 32(6):1222-1228. doi:10.1097/HJH.0000000000000175. [PubMed: 24786293]

44. Gorman BK, Sivaganesan A. The role of social support and integration for understanding socioeconomic disparities in self-rated health and hypertension. Soc Sci Med 1982. 2007; 65(5): 958-975. doi:10.1016/j.socscimed.2007.04.017.

45. Bell CN, Thorpe RJ, LaVeist TA. Race/Ethnicity and Hypertension: The Role of Social Support. Am J Hypertens. 2010; 23(5):534-540. doi:10.1038/ajh.2010.28. [PubMed: 20186126]

46. Freedman BI, Murea M. Target Organ Damage in African American Hypertension: Role of APOL1. Curr Hypertens Rep. 2012; 14(1):21-28. doi:10.1007/s11906-011-0237-4. [PubMed: 22068337]

47. Cooper RS, Wolf-Maier K, Luke A, et al. An international comparative study of blood pressure in populations of European vs. African descent. BMC Med. 2005; 3:2. doi:10.1186/1741-7015-3-2. [PubMed: 15629061]

48. Wise IA, Charchar FJ. Epigenetic Modifications in Essential Hypertension. Int J Mol Sci. 2016; 17(4) doi:10.3390/ijms17040451.

49. Teppala S, Shankar A, Ducatman A. The association between acculturation and hypertension in a multiethnic sample of US adults. J Am Soc Hypertens JASH. 2010; 4(5):236-243. doi:10.1016/ j.jash.2010.07.001. [PubMed: 20728423]

50. Centers for Disease Control and Prevention (CDC). Health status of American Indians compared with other racial/ethnic minority populations--selected states, 2001-2002. MMWR Morb Mortal Wkly Rep. 2003; 52(47):1148-1152. [PubMed: 14647016]

51. Kaholokula JK, Iwane MK, Nacapoy AH. Effects of Perceived Racism and Acculturation on Hypertension in Native Hawaiians. Hawaii Med J. 2010; 69(5 suppl 2):11-15. [PubMed: 20544603]

52. Schwingshackl L, Hoffmann G. Diet Quality as Assessed by the Healthy Eating Index, the Alternate Healthy Eating Index, the Dietary Approaches to Stop Hypertension Score, and Health Outcomes: A Systematic Review and Meta-Analysis of Cohort Studies. J Acad Nutr Diet. 2015; 115(5):780-800.e5. doi:10.1016/j.jand.2014.12.009. [PubMed: 25680825]

53. Brook RD, Appel LJ, Rubenfire M, et al. Beyond Medications and Diet: Alternative Approaches to Lowering Blood Pressure A Scientific Statement From the American Heart Association. Hypertension. 2013; 61(6):1360-1383. doi:10.1161/HYP.0b013e318293645f. [PubMed: 23608661]

54. Williams DR, Mohammed SA. Racism and Health II: A Needed Research Agenda for Effective Interventions. Am Behav Sci. 2013; 57(8) doi:10.1177/0002764213487341.

55. Rainforth MV, Schneider RH, Nidich SI, Gaylord-King C, Salerno JW, Anderson JW. Stress reduction programs in patients with elevated blood pressure: A systematic review and metaanalysis. Curr Hypertens Rep. 2008; 9(6):520-528. doi:10.1007/s11906-007-0094-3.

56. Ludwig J, Sanbonmatsu L, Gennetian L, et al. Neighborhoods, Obesity, and Diabetes - A Randomized Social Experiment. N Engl J Med. 2011; 365(16):1509-1519. doi:10.1056/ NEJMsa1103216. [PubMed: 22010917]

57. Lewis TT, Cogburn CD, Williams DR. Self-Reported Experiences of Discrimination and Health: Scientific Advances, Ongoing Controversies, and Emerging Issues. Annu Rev Clin Psychol. 2015; 11(1):407-440. doi:10.1146/annurev-clinpsy-032814-112728. [PubMed: 25581238] 


\section{KEY POINTS}

Hypertension is a leading cause of cardiovascular disease and stroke and this burden falls heavily on Blacks (or African Americans).

We review recent research on psychosocial factors and hypertension and attempt to contextualize the findings within a health disparities framework.

Our review reveals that psychosocial factors, such as socioeconomic status, stressors (including race-related stressors), and emotional states, may contribute to hypertension risks.

Future research should investigate how psychosocial factors accumulate over the life course to contribute to hypertension disparities.

Further research on psychosocial factors and hypertension can enhance the effectiveness of interventions to reduce hypertension risks in ethnic minority patients and communities. 


\section{SYNOPSIS}

Hypertension remains a leading contributor to cardiovascular disease and stroke. Despite advances in knowledge, awareness, and interventions Blacks (or African Americans) still have the highest prevalence of hypertension in the United States. Growing evidence suggests that psychosocial factors increase the risks for hypertension and help to account for racial differences in this condition. We review recent research on psychosocial factors and hypertension, and attempt to contextualize the findings within the framework of health disparities. Our review reveals that psychosocial factors, such as socioeconomic status, stressors, including race-related ones, emotional states, and social relationships all contribute to hypertension risks. However, our understanding remains limited about how these factors relate to each other and accumulate over the life course to contribute to hypertension disparities. Future research needs to give more concerted attention to identifying how research on psychosocial factors and hypertension can enhance the effectiveness of interventions to reduce hypertension risk in ethnic minority patients and communities. 